\title{
Mid-infrared imaging of the circumstellar dust around three Herbig Ae stars: HD 135344, CQ Tau, and HD 163296^
}

\author{
C. Doucet ${ }^{1}$, E. Pantin ${ }^{1}$, P. O. Lagage ${ }^{1}$, and C. P. Dullemond ${ }^{2}$ \\ 1 AIM, Unité Mixte de Recherche CEA - CNRS - Université Paris VII - UMR 7158, DSM/DAPNIA/Service d'Astrophysique, \\ CEA/Saclay, 91191 Gif-sur-Yvette, France \\ e-mail: doucetc@cea.fr \\ 2 Max-Planck-Institut fur Astronomie Heidelberg, Konigstuhl 17, Heidelberg, Germany
}

Received 18 October 2005 / Accepted 22 August 2006

\begin{abstract}
Aims. Planet formation has been known for many years to be tied to the spatial distribution of gas and dust in disks around young stars. To constrain planet formation models, imaging observations of protoplanetary disks are required.

Methods. Given this, we have undertaken a mid-infrared imaging survey of Herbig Ae stars, which are pre-main sequence stars of intermediate mass still surrounded by a large amount of circumstellar material. The observations were made at a wavelength of $20.5 \mu \mathrm{m}$ with the CAMIRAS camera mounted at the Cassegrain focus of the Canada France Hawaii Telescope.

Results. We report the observations of three stars, HD 135344, CQ Tau, and HD 163296. The circumstellar material around the three objects is spatially resolved. The extensions feature a disk-like shape. The images provide direct information on two key parameters of the disk: its inclination and its outer radius. The outer radius is found to be quite different from the one deduced from disk models, which is only constrained by fitting the Spectral Energy Distribution of the object. Other parameters of the disk, such as flaring and dust mass have been deduced from fitting both the observed extension and the spectral energy distribution with sophisticated disk models.

Conclusions. Our results show how important imaging data are to tighten constraints on the disk model parameters.
\end{abstract}

Key words. circumstellar matter - stars: formation - stars: pre-main-sequence - stars: individual: HD 163296 stars: individual: CQ Tau, stars: individual: HD 135344

\section{Introduction}

The formation of circumstellar disks is a natural outcome of the star formation process by which a molecular core collapses to form a star (Shu et al. 1987). Circumstellar disks can outlive the period during which stars form and still be present when the star is in its Pre-Main-Sequence (PMS) phase. In these disks, composed of gas and dust, various physical processes can lead to the growth of dust grains and eventually to the formation of planets. Understanding the physical conditions that prevail in these objects is of crucial importance when studying planet formation.

The study of circumstellar disks is a rapidly developing field, both from the observational and the modeling points of view (e.g., Natta 2004, and references there-in). The Infrared Space Observatory (ISO) has given clues about the dust composition of a sample of isolated HAeBe systems (Bouwman et al. 2001; Meeus et al. 2001). While these spectra reveal the composition of the dust, no direct information concerning the spatial distribution of the different dust species can be inferred from the ISO data. Most studies so far have used the Spectral Energy Distributions (SEDs) to put constraints on the spatial distribution of the circumstellar material.

* Based on observations obtained at the Canada France Hawaii Telescope (CFHT), which is operated by the national Research Council of Canada, the Institut National des Sciences de l'Univers of the Centre National de la Recherche Scientifique of France, and the University of Hawaii.
Models of protoplanetary disks are increasingly successful at accounting for much of the observed properties. For instance, they can justify that disks' SEDs are generally rather flat in $v F_{v}$, where $v$ is the frequency and $F_{v}$ the flux (Kenyon \& Hartmann 1987). Furthermore, models can explain that dust features are almost all seen in emission (Calvet et al. 1991; Chiang \& Goldreich 1997, hereafter CG97), reveal the presence of a near-infrared excess in the SEDs of Herbig Ae stars (Natta et al. 2001; Dullemond et al. 2001, hereafter DDN01), and interpret the differences observed in the far-IR excesses (Dullemond et al. 2002; Dullemond \& Dominik 2004). Fitting the SED only allows us to make conjectures about the disks' geometrical apparearance; spatially resolved imaging data of those disks are absolutely necessary to verify theories and models' assumptions. For instance, key parameters, such as the disk surface density profile with radius, are still very poorly constrained when fitting SEDs.

Mid-infrared imaging observations from a large groundbased telescope are potentially well suited to bringing spatial information on disks around Herbig Ae (HAe) stars. HAe stars represent the middle stage of PMS evolution of intermediatemass stars $\left(\sim 2-3 M_{\odot}\right)$; they are bright enough to heat submicron dust grains at $100 \mathrm{AU}$ to a temperature of about $150 \mathrm{~K}$. Grains at such a temperature have their peak of thermal emission in the mid-InfraRed (mid-IR). The diffraction limited angular resolution achievable with a $3.6 \mathrm{~m}$ class telescope in the mid-IR, $0.6 / 1.2 \operatorname{arcsec}$ at $10 / 20 \mu \mathrm{m}$, corresponds to a distance of $60 / 120 \mathrm{AU}$ for a star located at a typical distance of $100 \mathrm{pc}$, 
Table 1. Properties of the three Herbig Ae stars observed with the CAMIRAS mid-InfraRed camera. First two columns: coordinates of the objects (right ascension and declination); third column: their spectral type; fourth column: their distance, as deduced from Hipparcos data; fifth column: flux in the IRAS $25 \mu \mathrm{m}$ band; sixth column: flux at $20 \mu \mathrm{m}$ obtained in this paper; seventh column: age of the objects in Myr.

\begin{tabular}{lllcccccc}
\hline \hline Object & RA (2000) & Dec (2000) & Spectral type & Distance (pc) & $F_{25}(\mathrm{Jy})$ & $F_{20}(\mathrm{Jy})$ & Age (Myr) & References \\
\hline HD 135344 & 151548.4 & -370916 & F4V & $140_{-42}^{+42}$ & 6.7 & $5 \pm 1$ & $8 \pm 4$ & $1,2,4,8,10,11$ \\
HD 163296 & 175621.4 & -15720 & A3Ve & $122_{-16}^{+13}$ & 21 & $18 \pm 4$ & $7 \pm 5$ & $3,4,5,11,12$ \\
CQ Tau & 053558.4 & +244454 & A1-F5IVe & $100_{-17}^{+25}$ & 20.6 & $23 \pm 3$ & 10 & $6,7,9,12$ \\
\hline
\end{tabular}

References: (1) Coulson et al. (1998); (2) Dominik et al. (2003); (3) Jayawardhana et al. (2001); (4) Meeus et al. (2001); (5) Mannings \& Sargent (1997); (6) Mannings \& Sargent (2000); (7) Natta et al. (2001); (8) Sylvester et al. (1996); (9) Testi et al. (2001); (10) Thi et al. (2001); (11) van Boekel et al. (2005); (12) van den Ancker et al. (1998).

and thus allows for a relatively good sampling of disks whose sizes range up to several hundreds of AU. The first attempts to resolve the spatial structure of the circumstellar material around HAe stars were performed with multi-aperture observations with a single bolometer; emissions extending up to large distances from the star (more than 5 arcsec) were found around 3 objects HD 97048, HD 97300, and HD 176386 (Prusti et al. 1994). Such extended emissions were attributed to the emission from large molecules and small grains transiently heated by starlight and distributed in a dust shell with a large inner radius, probably a remnant from the cloud from which the star was born. It is only with the advent of mid-IR cameras that the full potential of mid-IR observations to study disk structures was achieved. Single dish observations have revealed the disk structure in the 500 AU (Astronomical Units) range around two HAe stars: AB Aur (Marsh et al. 1995; Pantin et al. 2005) and HD 100546 (Grady et al. 2001; Liu et al. 2003). Interferometric observations have allowed for the probing of the innermost regions of the disk (1-10 AU), and numerous objects have been observed (Millan-Gabet et al. 2001; Tuthill et al. 2002; Wilkin \& Akeson 2003; Leinert et al. 2004; Liu et al. 2005). Mid-IR single dish observations have now clearly demonstrated in a few examples that they can provide unique information on the disk structure, although the constraints brought on the disk modeling by such spatial information have not yet been fully exploited.

In this paper, we are interested in both increasing the number of HAe for which information on the mid-IR spatial extension is available, and in using this information, in combination with the already existing SED measurements between 1 and $100 \mu \mathrm{m}$, to constrain the parameters of the disk models developed these last years. The paper is organized as follows: the observations and data reduction are described in Sect. 2. Section 3 deals with the results, in terms of spatial extensions. In Sect. 4, a first, simple approach is used to establish the disk inclination, and in Sect. 5, we describe the 2-D radiative transfer code used to reproduce the observations. A discussion of the results follows in Sect. 6. Conclusions are drawn in Sect. 7.

\section{Observations and data reduction}

We have observed a sample of three Herbig Ae stars: HD 135344, CQ Tau, and HD 163296. Table 1 presents the main stellar parameters of the sample. The objects were selected from the catalogue of Thé et al. (1994) and Malfait et al. (1998) according to the following criteria: the objects are bright in the mid-IR, relatively close, and isolated, i.e., not associated with extended diffuse emission due to the parental cloud.

CQ Tau is located at a distance of $100_{-17}^{+25} \mathrm{pc}$ and has an age of 10 Myr (Natta et al. 2001; van den Ancker et al. 1998).
HD 163296 is at a distance of $122_{-16}^{+13} \mathrm{pc}$ and has an age of $7 \pm 5$ Myr (van Boekel et al. 2005). The distance and age of HD 135344 are more controversial. Until 2001, a distance of $84 \mathrm{pc}$ (Meeus et al. 2001) with an age of $17 \pm 3$ Myr (Thi et al. 2001) were used for this object. But, in a recent paper (van Boekel et al. 2005), the distance was re-evaluated to $140 \pm 42 \mathrm{pc}$ and the age to $8 \pm 4 \mathrm{Myr}$; these latter values will be used in the following.

The observations were performed with the mid-IR camera CAMIRAS (Lagage et al. 1992) installed as a visiting instrument at the Cassegrain focus of the Canada France Hawaii Telescope (CFHT). The camera is equipped with a Boeing $128 \times 128$ pixels Blocked Impurity Band (BIB) detector sensitive up to a wavelength, $\lambda$, of $\sim 27 \mu \mathrm{m}$. A filter centered at $20.5 \mu \mathrm{m}$ with a Full Width Half Maximum (FWHM) bandpass, $\Delta \lambda$, of $1.11 \mu \mathrm{m}$ was used. The Pixel Field of View (PFoV) on the sky was 0.29 arcsec; such a PFoV provides a good sampling of the diffraction pattern, which is of 1.5 arcsec FWHM.

The objects were observed between March 18, 2000 and March 24, 2000. During the run, seeing and weather conditions were extremely favorable and stable in time. HD 163296 and CQ Tau were observed at a median airmass of 1.4, and HD 135344 at an airmass around 1.9, which is the best achievable when observing from CFHT. Standard chopping and nodding techniques were applied to suppress atmosphere and telescope background emissions; the chopping throw was 16 arcsec to the North and the frequency used was $3.33 \mathrm{~Hz}$; the nodding amplitude was 20 arcsec to the West. The nodding direction was perpendicular to the chopping direction, to get the best spatial resolution; given the low chopping and nodding throw and the field of view of the camera, the source always remained within the detector field of view and the obtained images thus contain 4 beams ( 2 positive, 2 negative). Given the huge photon background in the mid-IR, the elementary integration time was set to $15 \mathrm{~ms}$, and the images were co-added in real time to store only two co-added images (one for each chopping position) every second.

The basic data reduction is standard. The data cubes of one observation are carefully stacked with the rejection of corrupted planes. A shift-and-add procedure is applied to each cube of images using a correlation based method with a re-sampling factor of $8: 1$. The four beams are then combined in one image by a source extraction algorithm followed by a shift-and-add procedure. Finally, flux calibration is achieved via aperture photometry of a set of photometric standard stars such as $\alpha$ Tau, $\alpha$ Boo, $\beta \mathrm{Gem}$, or $\gamma$ Dra (Cohen et al. 1999). The photometry gives a total flux of $6.3 \pm 0.6 \mathrm{Jy}$ for HD 135344, $23 \pm 3 \mathrm{Jy}$ for CQ Tau, and $18 \pm 4$ Jy for HD 163296, in good agreement with the IRAS values (Table 1). 

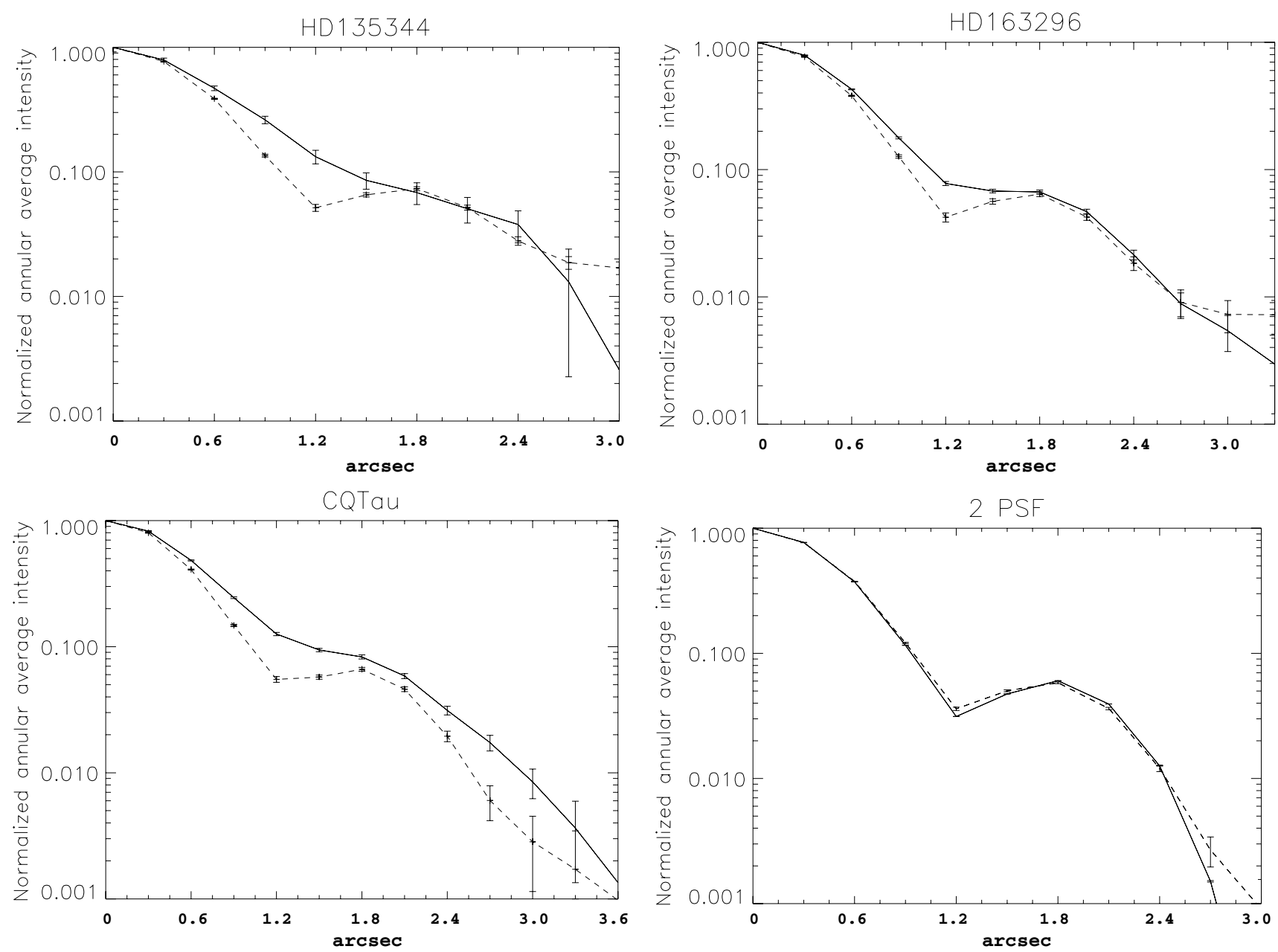

Fig. 1. Annular averages intensity aperture for the source (solid line) and PSF (dashed line) normalized to the peak value, as a function of radius. The errors drawn are $1 \sigma$-errors rms. The reference star for HD 135344 is $\alpha$ Boo, for HD $163296 \gamma$ Dra, and for CQ Tau, $\beta$ Gem. The plot labeled "2 PSF" shows two reference stars, $\alpha$ Boo and $\gamma$ Dra, as observed on the March 24, 2000. Although the two reference stars have very different fluxes ( $\gamma$ Dra with 43 Jy and $\alpha$ Boo with 170 Jy, Van Malderen et al. 2004), the two profiles are similar.

\section{Extended emission}

The three objects of the sample are spatially resolved. This can be seen in Fig. 1 where we have compared the average annular profile of the object with those of the Point Spread Function (PSF), obtained from the observation of a reference point-like star. Extended emission is detected up to 100-300 AU.

We carefully checked that the observed extensions are not artifacts, but the result of true extended emission from the objects. Several arguments lead us to reject explanations of the extensions in terms of temporal variations of the PSF between the observation of the object and the observation of the reference star. One possible cause of such temporal PSF variations could be variations of the seeing. This hypothesis is however rejected for the following reasons. First, concerning the limitations of the spatial resolution, the seeing contribution at $20.5 \mu \mathrm{m}$ is negligible with respect to the diffraction: for a typical seeing value of $0.8^{\prime \prime}$ FWHM in the visible range, one can estimate a seeing contribution at $20.5 \mu \mathrm{m}$ around $0.4^{\prime \prime}$, when using the $\lambda^{(-1 / 5)}$ scaling law; thus, seeing induces PSF changes of the order of 1 pixel FWHM, which is much smaller than the widths of observed extensions. Secondly, the seeing was quite stable during the observations; thus we estimated seeing variation effects to be much lower than one pixel. Note also that two of the objects were observed during different nights. HD 135344 was observed for four different nights: on March 18, 2000 (exposure time 6 mn), on March 19 (exposure time: $3 \mathrm{mn}$ ), on March 21 (exposure time: $6 \mathrm{mn}$ ), and on March 24, (exposure time: $2 \mathrm{mn} 30$ ). Its extension is confirmed over the 4 nights. CQ Tau was observed during 2 different nights on March 20, and on March 21 (respectively, with 3 and $6 \mathrm{mn}$ of integration time) and is spatially extended in both datasets. HD 163296 was observed only once (exposure time: $3 \mathrm{mn}$ ) on March 24.

Another possible source of fake extensions could be chromatic effects. Indeed, HAe stars have large infrared excesses, and thus have SEDs quite different from that of the PSF reference stars. Any filter leak, either on the blue or on the red side of the nominal filter bandpass, would then potentially lead to PSF variations between point-like HAe objects and PSF reference stars. We double-checked the filter transmission at the operating temperature of the filter $(10 \mathrm{~K})$ using a Fourier Transform Spectrometer with a spectral resolution of $4 \mathrm{~cm}^{-1}$. The rejection rate outside the filter bandpass is typically better than $10^{-3}$. We simulated the PSF variations due to such a filter using the mid-IR spectra of the objects obtained with ISO (Meeus et al. 2001) for two of the three extended objects; (CQ Tau was not observed by ISO SWS). The simulated PSF variations lead to some extended emission, but much fainter than observed, both in intensity and in spatial extension. Thus, an explanation of the 


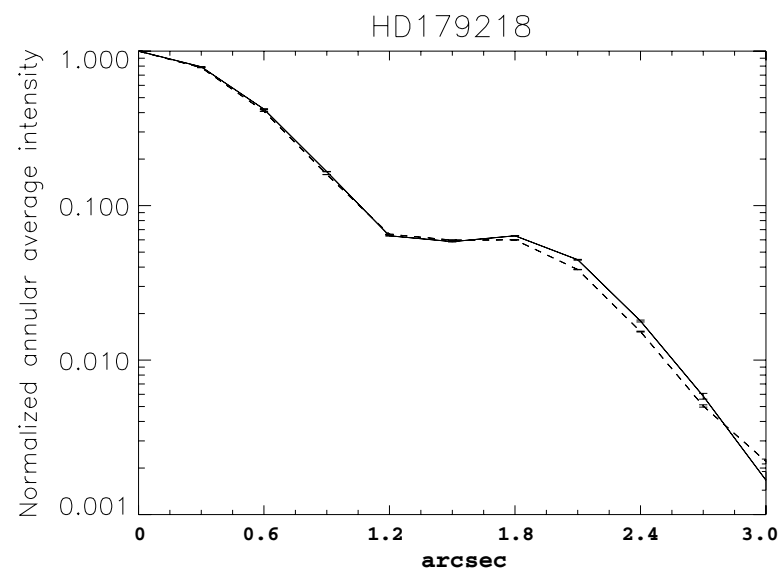

Fig. 2. Same as Fig. 1, to make a comparison with HD 179218 for which there is no extended emission detected.

observed extensions in terms of chromatic variations of the PSF can be discarded.

Note also that not all the HAe stars observed are extended. To make a comparison, a far away HAe star, with a similar (even greater) IR excess, HD 179218 located at $240 \mathrm{pc}$ (Meeus et al. 2001), was observed. This object does not show extended emission at $20.5 \mu \mathrm{m}$ (Fig. 2). This is an additional argument to conclude that the extension observed in HD 135344, CQ Tau, and HD 163296 is really due to an extended emission from the objects.

\section{Inclination of the disk}

Extensions around two out of the three objects, namely CQ Tau and HD 163296, were already observed at other wavelengths. Testi et al. (2001) have resolved the emission around CQ Tau at $7 \mathrm{~mm}$ and concluded that it was compatible with a disk-like geometry. Grady et al. (2001) have obtained coronographic images of HD 163296 with the Space Telescope Imaging Spectrograph on board the Hubble Space Telescope, which revealed a circumstellar disk with a radius of 450 AU. Therefore, our modeling of the extensions seen in the mid-IR range assumes a priori a disk-like geometry.

Disks inclinations can be relatively easily determined if their emissions are spatially resolved. However, HAe disks are generally dominated in the mid-IR by the innermost regions (1-30 AU). Our goal was to detect the emission from the intermediate regions of the disks (30-200 AU). The disk emission can be decomposed, in our image, into a central unresolved component plus an extended one (whose geometry should reflect the true disk geometry at the distance scales achievable for our data). We first removed the "point-like" central emission component by subtracting a scaled PSF from the image of the object. The parameters of the point-like component (intensity, position) were computed automatically using a penalty functional (to avoid any visual bias) and then cross-checked visually using a dedicated graphical interface built in IDL. The resulting image, called residuals, is free from the central emission, so that the extended emission is enhanced and it is easier to determine the disk geometry. For each target, this processing was done using all available PSF measurements. The errors on the putative extensions were assessed when applying the same procedure to two PSF reference stars and, when possible, when comparing the extensions obtained from different nights. The results are shown in Figs. 6-8.
Table 2. Parameters of the elliptical fit of the image. In the first column, the inclination of the disk (0 degree means a faced-on disk). The position angle (PA) of the disk on the sky is measured counter-clockwise from the North. The distance corresponds to the maximum distance at which the disk is detected in the $20 \mu \mathrm{m}$ observations. The brightness level in the last column corresponds to the brightness of the maximum distance at which the disk is detected here.

\begin{tabular}{lcccc}
\hline \hline & $\begin{array}{c}i \\
(\mathrm{deg})\end{array}$ & $\begin{array}{c}\text { PA } \\
(\mathrm{deg})\end{array}$ & $\begin{array}{c}\text { Distance } \\
(\mathrm{AU})\end{array}$ & $\begin{array}{c}\text { Brightness level } \\
\left(\mathrm{mJy} /{ }^{\prime \prime 2}\right)\end{array}$ \\
\hline HD 135344 & $46 \pm 5$ & $100 \pm 10$ & 210 & 170 \\
HD 163296 & $60 \pm 5$ & $105 \pm 10$ & 215 & 135 \\
CQ Tau & $33 \pm 5$ & $120 \pm 10$ & 290 & 129 \\
\hline
\end{tabular}

The first result is the elliptical shape of the extensions, which is characteristic of the emission of disks inclined with respect to the line of sight. An ellipse fit of the residuals gives an estimate of the disks' inclinations and position angles. The results in terms of inclination and position angles are shown in Table 2.

The inclination of $33^{\circ} \pm 5$ found here for CQ Tau is in the middle range of values found in the literature, which range from $63^{\circ}$ (Testi et al. 2001) to $14^{\circ}$ (Dent et al. 2005); in between we can find $48_{-3^{\circ}}^{\circ}\left(\mathrm{PA}=105^{\circ} \pm 5\right)$ (Eisner et al. 2004).

For HD 163296 , the disk inclination of $60^{\circ} \pm 5^{\circ}$ is in good agreement with that found by Mannings \& Sargent (1997) of $58^{\circ}$. Concerning the position angle of the disk, Mannings \& Sargent (1997) found $126^{\circ} \pm 3$ with CO observations, and Grady et al. (2000) found $140^{\circ} \pm 5$ thanks to optical coronographic images. We found a value of $105^{\circ} \pm 10$ when fitting our mid-IR data. This difference in the position angle could be related to the fact that our data are only sensitive to warm dust whose geometry could slightly differ from that seen at shorter or longer wavelengths, if the disk contains, for instance, non axisymmetric structures.

\section{Modeling}

We used a relatively simple parameterized model to investigate the dependence of the emission in the mid-IR on each parameter. We consider disks heated by irradiation from the central star. The density profile of the gas is parameterized as a function of $r$ (radius) and $z$ (vertical height above the disk mid-plane):

$\rho(r, z)=\frac{\Sigma(r)}{\sqrt{2 \pi} H_{p}(r)} \exp \left(-\frac{z^{2}}{2 H_{p}(r)^{2}}\right)$

and it is assumed that the dust is well mixed with the gas. The surface density is assumed to follow a power-law in radius: $\Sigma(r)=\Sigma_{0}\left(r / r_{0}\right)^{-p}$, with $r_{0}$ a fiducial radius. The scale height of the disk $H_{p}(r)$ is also assumed to be a power-law: $H_{p}(r)=H_{0}\left(r / r_{0}\right)^{q}$. The inner radius of the disk $\left(r=R_{\text {in }}\right)$ is located at the dust evaporation radius $(1400-1500 \mathrm{~K}$ for silicate dust). The inner boundary (rim) is directly exposed to the stellar flux and is puffed up since it is hotter than the rest of the disk. Here, we mimic the puffing-up of the rim predicted by DDN01 by a specified value of $H_{p \text {,in }}$ at $r=R_{\text {in }}$, which is a parameter of the model. It should be noted that whether such an inner rim is indeed puffed-up is still a matter of debate. Moreover, Isella $\&$ Natta (2005) have shown that the rim is probably rounded-off due to the density-dependence of the dust sublimation temperature. This effect is not included here.

For the dust opacities, we use those of Draine \& Li (2001). We use a MRN (Mathis et al. 1977) distribution of grains 

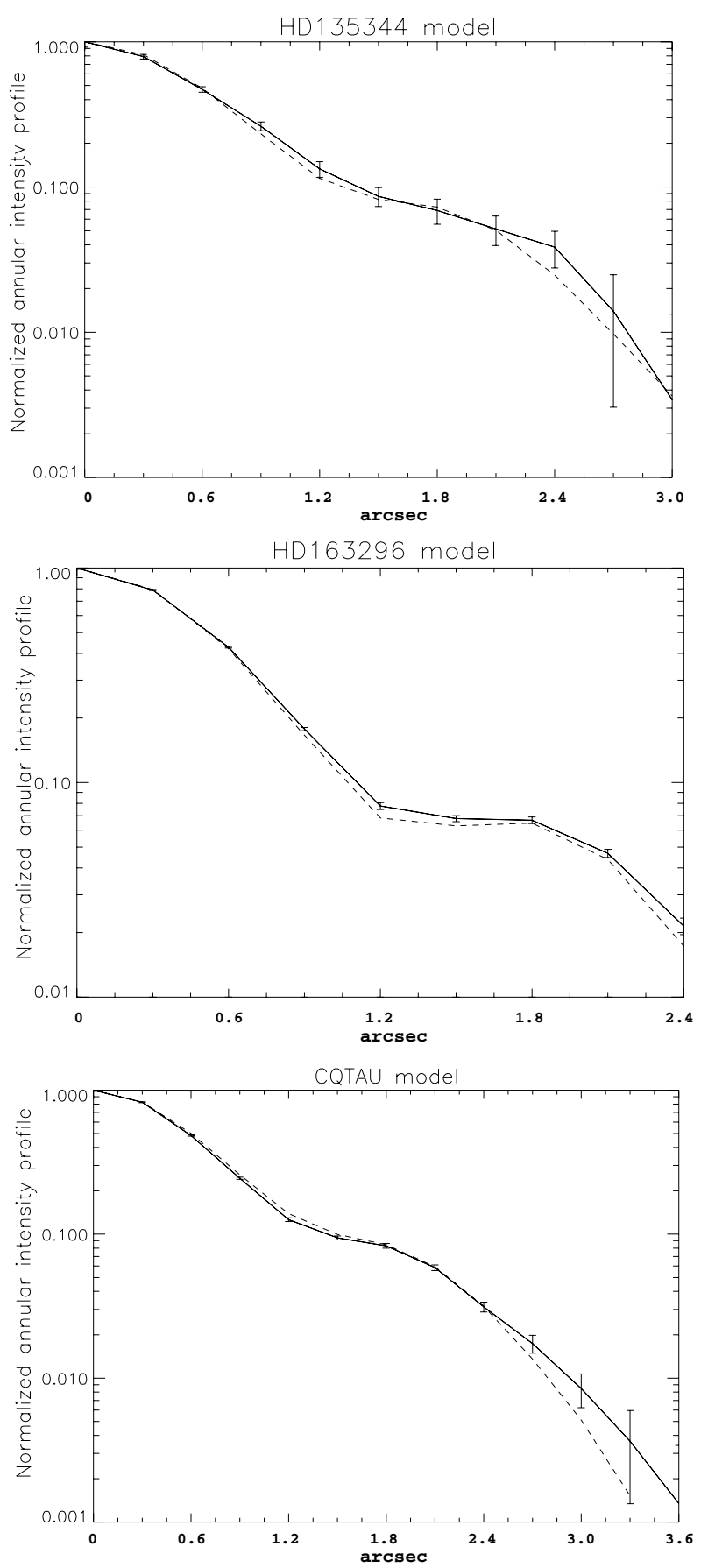

Fig. 3. Annular average intensity aperture normalized to the peak for the observations (full line), model (dashed line), and the PSF associated (dot-dashed line). We have constructed an image with the DD04 model that we have convolved with the PSF of the associated night. The error in the profile comes from the noise rms in the image.

$\left(n(a) \propto a^{-3.5}\right)$ with a size between 0.01 and $0.3 \mu \mathrm{m}$. It is the disk surface layer that dominates the SED in the mid-IR range. The emission of the surface is made by small grains that trace the disk geometry. We will focus on this component in this paper. Since the objects have no PAH (Polycyclic Aromatic Hydrocarbon) emission (or only a weak one in the case of HD 135344), we do not take into account this population of grains.

Once the density profile is set, and the dust opacities and stellar parameters are given, the code RADMC (Dullemond \& Dominik 2004; Pontoppidan \& Dullemond 2005) solves the
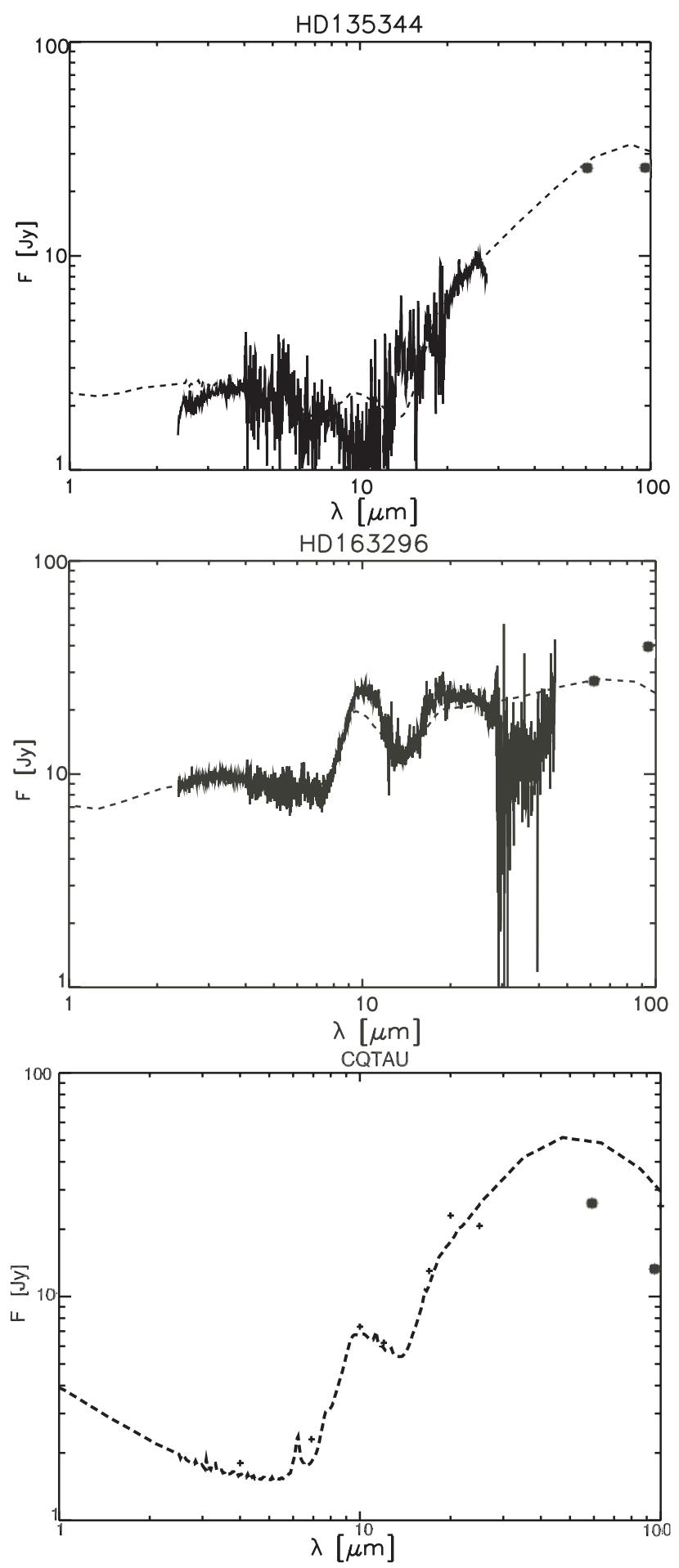

Fig. 4. Modeled spectrum (dashed line) between 1 and $100 \mu \mathrm{m}$. For HD 135344 and HD 163296, the ISO spectrum is overplotted by a bold line. For CQ Tau, there are some photometric points from Grady et al. (2005); we have added the one from this study at $20.5 \mu \mathrm{m}$ and IRAS points at 60 and $100 \mu \mathrm{m}$ (filled circles).

temperature structure of the disk with a Monte-Carlo method using a variant of the algorithm of Bjorkman \& Wood (1997). This Monte-Carlo code also produces the source terms for scattering, in the isotropic-scattering approximation. With a ray-tracing tool (which is part of the code RADICAL, see Dullemond \& Turolla (2000) for a detailed description) the SED and images can then be produced and compared to the observations. Comparative 

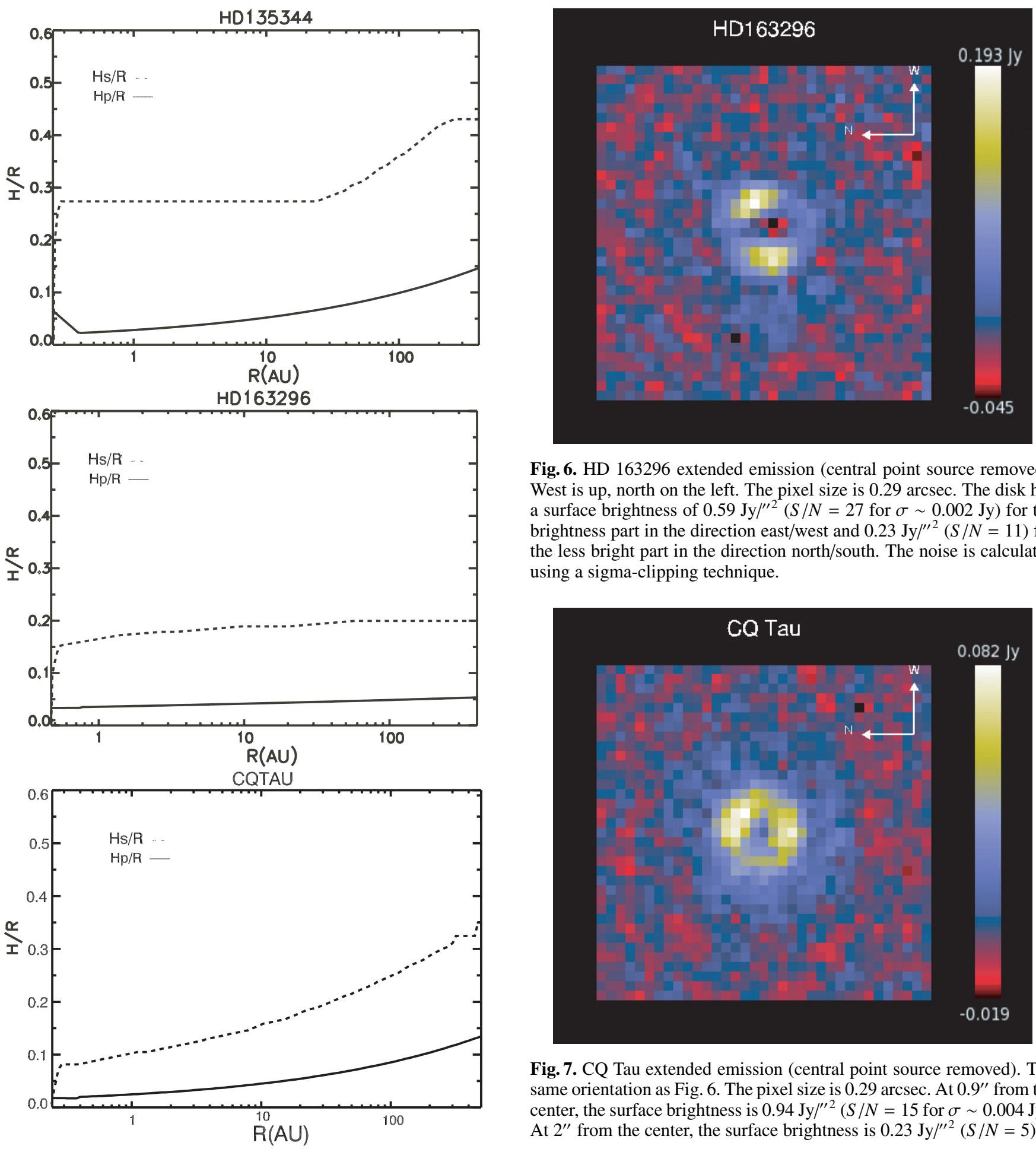

Fig. 6. HD 163296 extended emission (central point source removed). West is up, north on the left. The pixel size is $0.29 \operatorname{arcsec}$. The disk has a surface brightness of $0.59 \mathrm{Jy} /{ }^{\prime 2}(S / N=27$ for $\sigma \sim 0.002 \mathrm{Jy})$ for the brightness part in the direction east/west and $0.23 \mathrm{Jy} /{ }^{\prime 2}(S / N=11)$ for the less bright part in the direction north/south. The noise is calculated using a sigma-clipping technique.

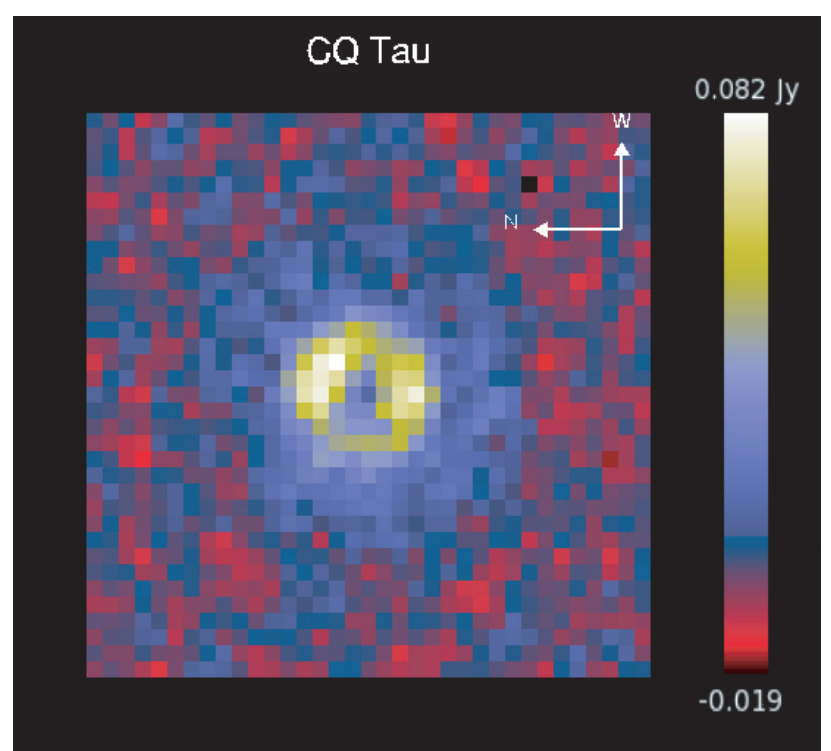

Fig. 7. CQ Tau extended emission (central point source removed). The same orientation as Fig. 6. The pixel size is 0.29 arcsec. At $0.9^{\prime \prime}$ from the center, the surface brightness is $0.94 \mathrm{Jy} /{ }^{\prime \prime 2}(S / N=15$ for $\sigma \sim 0.004 \mathrm{Jy})$. At $2^{\prime \prime}$ from the center, the surface brightness is $0.23 \mathrm{Jy} /{ }^{\prime \prime 2}(S / N=5)$.

Fig. 5. Modelized vertical structure versus the radius. The pressure height scale $\left(H_{p} / R\right)$ is the solid line and the surface height scale (i.e., the height of the disk photosphere above the mid-plane $H_{s} / R$ ) is the dashed line.

images are obtained by first resampling the maps to the CAMIRAS sampling and then by convolving them with the PSF.

\section{Comparison model versus observation}

For each object, the best model should simultaneously fit the SED (Fig. 4) and the extension found at $20.5 \mu \mathrm{m}$ with our observations (Fig. 3). Concerning HD 135344 and HD 163296, we

used mainly the ISO spectrum to constrain the SED; as far as CQ Tau is concerned, IRAS photometry and BASS points obtained by Grady et al. (2005) are used. Figure 5 shows the structure of the disk in terms of pressure and surface scale height.

Multiple runs of the model are performed until a satisfactory fit to the observed spectrum and the extension found at $20.5 \mu \mathrm{m}$ is obtained. In the fitting procedure, the stellar parameters ( $T_{\text {eff }}, M_{\star}, R_{\star}$, see Table 3 ), the dust evaporation temperature (i.e., the position of the inner rim) at $1400 \mathrm{~K}$, the outer radius and the inclination of the disk, and the dust composition and size distribution are fixed. Other parameters, such as the pressure scale height for the inner rim (possibly puffed-up), the outer 


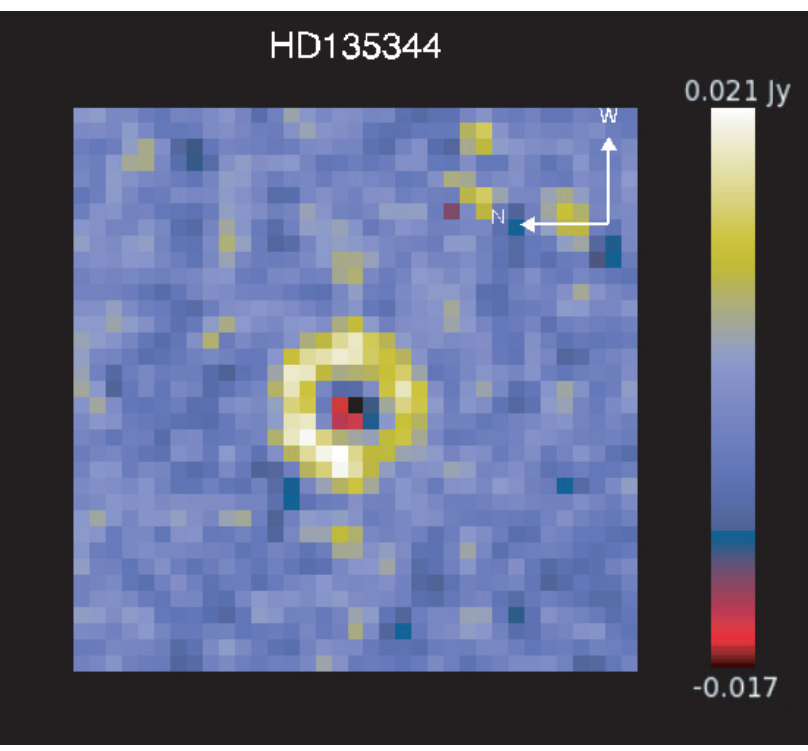

Fig. 8. HD 135344 extended emission (central point source removed). The same orientation as Fig. 6. The pixel size is 0.29 arcsec. At $0.9^{\prime \prime}$ from the center, the surface brightness is $0.25 \mathrm{Jy} / /^{\prime 2}(S / N=15$ for $\sigma \sim 0.0015 \mathrm{Jy}$ ).

pressure scale height $\left(H_{0}\right)$, the power-law index of the pressure scale height (i.e., $q$, which has been fixed in the case of flared disk to $9 / 7$, a value determinated by hydrostatic equilibrium, Chiang \& Goldreich 1997), the mass of the disk, and the powerlaw index of the surface density (i.e., $p$ ) are estimated. Fitting the SED gives one solution among several degenerate combinations. A minimum value of the outer radius is derived from $20 \mu \mathrm{m}$ observations, which is a very strong constraint on the true disk size, thus largely removing the degeneracy of the set of model parameters.

As first guesses, we used those parameters found when fitting the SED by Dominik et al. (2003) for HD 135344 and HD 163296, and those by Chiang et al. (2001) for CQ Tau. Trying to fit the SED, we focused mainly on the Near-IR and mid-IR regions. Indeed, those regions are the regions where most of the reprocessed stellar energy (by the disk's surface layer) emerged, and therefore are the most strongly affected by the model and the geometry of the disk. Not all the parameters are sensitive to the spatial distribution at $20.5 \mu \mathrm{m}$ (Table 4). The CAMIRAS images put a strong constraint on the minimum outer radius, and we have to find a solution with all the free parameters to reproduce the shape of the SED, the extension and the total flux at $20.5 \mu \mathrm{m}$.

For HD 135344, the disk parameters deduced from fitting only the SED of HD 135344 were close to those that allow us to fit both the SED and our observations. For CQ Tau, in addition to previous studies fitting only the SED, we had to take into account the fact that the disk is quite extended at $20 \mu \mathrm{m}$ (about $300 \mathrm{AU}$ ) and is observed with an inclination of $33^{\circ} \pm 5$. The fast increase of the disk emission in the [10-30] $\mu \mathrm{m}$ range can be modeled only with a flared disk (with $H_{p}^{\text {out }} / R_{\text {out }}>0.1$ ). In the framework of our modeling, we found that the only manner to simultaneously obtain a quite low far-IR excess (as seen in the spectrum) with the observed extended $20 \mu \mathrm{m}$ emission is a low-mass disk of only $0.005 M_{\odot}$ with a pressure scale height of $58 \mathrm{AU}$ at $450 \mathrm{AU}$. The total flux in the infrared excess compared to the stellar flux is determinated by the fraction of the central star energy intercepted by the disk. This covering fraction is linked to $H_{\mathrm{p}}^{\text {out }} / R_{\text {out }}$, where the disk geometry thickness is maximum. Here, this parameter is the same as the previous study (energy conservation), but for a different outer radius. That means that the disk is less flared than deduced earlier. For CQ Tau we obtain a disk mass 10 times smaller than that already found (Testi et al. 2003; Chiang et al. 2001), and this mass only traces the small grains. The disk emission at $\mathrm{mm}$ wavelength is determined by the mid-plane grain and disk properties, and it is not affected by the nature of the surface dust. Consequently, in this paper, we have not tried to select the best parameters for the mid-plane dust to fit $\mathrm{mm}$ observations. Our underestimated flux at $\mathrm{mm}$ wavelength suggests that there must be big grains in the disk mid-plane of CQ Tau to recover the measured flux at these wavelengths (Testi et al. 2003).

HD 163296 is classified as a group II object (interpreted as being surrounded by a flat or self-shadowed disk); it should in principle be much more difficult to resolve the disk in the mid-IR range. Surprisingly, some extended mid-IR emission at $20 \mu \mathrm{m}$, although less prominent than for group I objects, is however observed. In the modeling, we used the parameter found in Dominik et al. (2003), who modeled the SED with a flared disk cut at $50 \mathrm{AU}$, as a first guess. Here, we modified the largescale pressure scale-height to mimic a weakly flared disk. The SED is well reproduced with a disk having little flaring (Fig. 4 and Table 3), while the extension at $20 \mu \mathrm{m}$ (Fig. 3) constrains the disk to have a minimum outer radius of $200 \mathrm{AU}$ with a disk mass of $0.01 M_{\odot}$.

\section{Conclusions and future work}

We have shown the strength of mid-IR imaging to constrain the disk properties. There are still a very limited number of objects with spatially resolved extended emission, and it is not yet possible to draw statistical conclusions about the spatial structure of disk around HAe stars. With the advance of mid-IR instruments on 8-m class telescopes, such as the VISIR (Lagage et al. 2004) instrument available on the MELIPAL Very Large Telescope (VLT) at the European Southern Observatory (ESO), higher angular resolution will be available and the field will further develop; more quantitative studies will be possible and the goal of retrieving detailed disk surface density profiles from the observations should be achievable.

Observations should not be limited to the $20 \mu \mathrm{m}$ atmospheric window; observations in the $10 \mu \mathrm{m}$ atmospheric window are also very promising, especially for those HAe stars whose spectra feature the so-called PAH bands' features at 3.3, 6.2, 7.7, 8.6, $11.2,12.7 \mu \mathrm{m}$. PAH bands are attributed to vibrational relaxation of UV-pumped Polycyclic Aromatic Hydrocarbon molecules containing about 50-100 carbon atoms (Allamandola et al. 1989; Puget \& Leger 1989). Their emission, as a function of the distance $r$ to the star, drops with a $r^{-2}$ power-law, much slower than thermal emission from large grains in thermal equilibrium. PAH's emission is thus a promising probe for studying flaring disks at large distances from the star, with the good angular resolution now achieved on large ground-based telescopes at 10 microns. One clear method of improving the field over the next few years would be to use sophisticated models to fit a combination of interferometric and single dish observations in the near- and mid-IR, which probe the innermost and intermediate disk regions, respectively.

Acknowledgements. We are gratefully indebted to P. Masse, R. Jouan, and M. Lortholary for their assistance with the CAMIRAS instrument, A. Claret in efficiently supporting us in our observations, as well as to the staff of CFHT/Hawaii 
Table 3. Stellar properties and fit parameters. The stellar parameters of CQ Tau are taken in Chiang et al. (2001). For HD 135344 and HD 163296, the parameters are taken from Meeus et al. (2001) and van Boekel et al. (2005). $H_{p}^{\text {in }} / R_{\text {in }}$ characterizes the puffed inner rim. $q$ is the power-law index of the scale height and $p$ that of the surface density. $H_{\mathrm{p}}^{\text {out }} / R_{\text {out }}$ characterizes the flaring angle of the disk. The last column is the flux at $20.5 \mu \mathrm{m}$ calculated from the modeled image.

\begin{tabular}{lcccccccccccc}
\hline \hline Object & $\begin{array}{c}\text { Distance } \\
(\mathrm{pc})\end{array}$ & $\begin{array}{c}M_{\star} \\
\left(M_{\odot}\right)\end{array}$ & $\begin{array}{c}T_{\text {eff }} \\
(\mathrm{K})\end{array}$ & $\begin{array}{c}R_{\star} \\
\left(R_{\odot}\right)\end{array}$ & $\begin{array}{c}R_{\text {in }} \\
(\mathrm{AU})\end{array}$ & $\begin{array}{c}H_{p}^{\text {in }} / R_{\text {in }} \\
R_{\text {out }} \\
(\mathrm{AU})\end{array}$ & $H_{p}^{\text {out }} / R_{\text {out }}$ & $p$ & $q$ & $\begin{array}{c}i \\
(\text { degrees })\end{array}$ & $\begin{array}{c}F(20.5 \mu \mathrm{m}) \\
(\mathrm{Jy})\end{array}$ \\
\hline $\begin{array}{l}\text { HD 135344 } \\
\text { (DDN03) }\end{array}$ & 84 & 1.3 & 6750 & 2.1 & 0.24 & 0.065 & 800 & 0.21 & 0.8 & $9 / 7$ & 60 & - \\
$\begin{array}{l}\text { HD 135344 } \\
\text { (in this study) }\end{array}$ & 140 & 1.3 & 6750 & 2.1 & 0.24 & 0.065 & 200 & 0.12 & 0.8 & $9 / 7$ & 45 & 5.7 \\
\hline $\begin{array}{l}\text { HD 163296 } \\
\text { (DDN03) }\end{array}$ & 122 & 2.5 & 10500 & 1.7 & 0.45 & 0.033 & 50 & 0.07 & 0.2 & $9 / 7$ & 65 & - \\
$\begin{array}{l}\text { HD 163296 } \\
\text { (in this study) }\end{array}$ & 100 & 2.5 & 10500 & 1.7 & 0.45 & 0.033 & 200 & 0.05 & 1. & 1.07 & 60 & 16.6 \\
\hline $\begin{array}{l}\text { CQ Tau } \\
\text { (Chiang2001) }\end{array}$ & 100 & 1.7 & 7130 & 1.3 & 0.23 & 0.0002 & 180 & 0.13 & 1.5 & $9 / 7$ & - & - \\
$\begin{array}{l}\text { CQ Tau } \\
\text { (in this study) }\end{array}$ & 100 & 1.7 & 7130 & 1.9 & 0.23 & 0.018 & 450 & 0.13 & 0.3 & $9 / 7$ & 33 & 20 \\
\hline
\end{tabular}

Table 4. Effects of the free parameters of the model on the SED and spatial distribution of the $20.5 \mu \mathrm{m}$ emission in the CAMIRAS image.

\begin{tabular}{|c|c|c|}
\hline Parameter & Influence on SED & Spatial distribution at $20.5 \mu \mathrm{m}$ \\
\hline$\Sigma_{0}$ & $\begin{array}{l}\text { Influence on the total flux in the mid-IR } \\
\text { and more strongly for } \lambda \geq 60 \mu \mathrm{m}\end{array}$ & No influence \\
\hline$p$ & No influence in the mid-IR & Little influence \\
\hline$H_{p}^{\mathrm{in}} / R_{\mathrm{in}}$ & $\begin{array}{l}\text { Modifies contrast mid-/near-IR } \\
\text { (structure inner rim/shadow) }\end{array}$ & $\begin{array}{l}\text { No influence } \\
\text { (inner rim }+ \text { shadow in the first pixel) }\end{array}$ \\
\hline$q$ & Modifies the whole shape of the SED & Large influence \\
\hline$H_{p}^{\text {out }} / R_{\text {out }}$ & Modifies flux in the mid- and far-IR & Little influence \\
\hline$R_{\text {out }}$ & Modifies far-IR emission & Large influence \\
\hline
\end{tabular}

for their support during the observing runs. CD wishes to thank J. Bouwman and E. Habart for very helpful discussions and advice.

\section{References}

Allamandola, L. J., Tielens, G. G. M., \& Barker, J. R. 1989, ApJS, 71, 733 Bouwman, J., Meeus, G., de Koter, A., et al. 2001, A\&A, 375, 950

Calvet, N., Patino, A., Magris, G. C., \& D’Alessio, P. 1991, ApJ, 380, 617

Chiang, E. I., \& Goldreich, P. 1997, ApJ, 490, 368

Chiang, E. I., Joung, M. K., Creech-Eakman, M. J., et al. 2001, ApJ, 547, 1077

Cohen, M., Walker, R. G., Carter, B., et al. 1999, AJ, 117, 1864

Coulson, I. M., Walther, D. M., \& Dent, W. R. F. 1998, MNRAS, 296, 934

Dent, W. R. F., Greaves, J. S., \& Coulson, I. M. 2005, MNRAS, 359, 663

Dominik, C., Dullemond, C. P., Waters, L. B. F. M., \& Walch, S. 2003, A\&A, 398, 607

Draine, B. T., \& Li, A. 2001, ApJ, 551, 807

Dullemond, C. P., \& Turolla, R. 2000, A\&A, 360, 1187

Dullemond, C. P., \& Dominik, C. 2004, A\&A, 417, 159

Dullemond, C. P., van Zadelhoff, G. J., \& Natta, A. 2002, A\&A, 389, 464

Eisner, J. A., Lane, B. F., Hillenbrand, L. A., Akeson, R. L., \& Sargent, A. I. 2004, ApJ, 613, 1049

Grady, C. A., Devine, D., Woodgate, B., et al. 2000, ApJ, 544, 895

Grady, C. A., Polomski, E. F., Henning, T., et al. 2001, AJ, 122, 3396

Grady, C. A., Woodgate, B. E., Bowers, C. W., et al. 2005, ApJ, 630, 958

Isella, A., \& Natta, A. 2005, A\&A, 438, 899

Jayawardhana, R., Fisher, R. S., Telesco, C. M., et al. 2001, AJ, 122, 2047

Kenyon, S. J., \& Hartmann, L. 1987, ApJ, 323, 714

Lagage, P. O., Jouan, R., Masse, P., Mestreau, P., \& Tarrius, A. 1992, in Progress in Telescope and Instrumentation Technologies, ed. M.-H. Ulrich, 601

Lagage, P. O., Pel, J. W., Authier, M., et al. 2004, The Messenger, 117, 12
Leinert, C., van Boekel, R., Waters, L. B. F. M., et al. 2004, A\&A, 423, 537 Liu, W. M., Hinz, P. M., Meyer, M. R., et al. 2003, ApJ, 598, L111 Liu, W. M., Hinz, P. M., Hoffmann, W. F., et al. 2005, ApJ, 618, L133 Malfait, K., Bogaert, E., \& Waelkens, C. 1998, A\&A, 331, 211

Mannings, V., \& Sargent, A. I. 1997, ApJ, 490, 792

Mannings, V., \& Sargent, A. I. 2000, ApJ, 529, 391

Marsh, K. A., Van Cleve, J. E., Mahoney, M. J., Hayward, T. L., \& Houck, J. R. 1995, ApJ, 451, 777

Mathis, J. S., Rumpl, W., \& Nordsieck, K. H. 1977, ApJ, 217, 425

Meeus, G., Waters, L. B. F. M., Bouwman, J., et al. 2001, A\&A, 365, 476

Millan-Gabet, R., Schloerb, F. P., \& Traub, W. A. 2001, ApJ, 546, 358

Natta, A., Prusti, T., Neri, R., et al. 2001, A\&A, 371, 186

Pantin, E., Bouwman, J., \& Lagage, P. O. 2005, A\&A, 437, 525

Pontoppidan, K. M., \& Dullemond, C. P. 2005, A\&A, 435, 595

Prusti, T., Natta, A., \& Palla, F. 1994, A\&A, 292, 593

Puget, J. L., \& Leger, A. 1989, ARA\&A, 27, 161

Shu, F. H., Lizano, S., \& Adams, F. C. 1987, in Star Forming Regions, ed. M. Peimbert, \& J. Jugaku, IAU Symp., 115, 417

Sylvester, R. J., Skinner, C. J., Barlow, M. J., \& Mannings, V. 1996, MNRAS, 279,915

Testi, L., Natta, A., Shepherd, D. S., \& Wilner, D. J. 2001, ApJ, 554, 1087

Testi, L., Natta, A., Shepherd, D. S., \& Wilner, D. J. 2003, A\&A, 403, 323

Thé, P. S., de Winter, D., \& Perez, M. R. 1994, A\&AS, 104, 315

Thi, W. F., van Dishoeck, E. F., Blake, G. A., et al. 2001, ApJ, 561, 1074

Tuthill, P. G., Monnier, J. D., Danchi, W. C., Hale, D. D. S., \& Townes, C. H. 2002, ApJ, 577, 826

van Boekel, R., Min, M., Waters, L. B. F. M., et al. 2005, A\&A, 437, 189

van den Ancker, M. E., de Winter, D., \& Tjin A Djie, H. R. E. 1998, A\&A, 330, 145

Van Malderen, R., Decin, L., Kester, D., et al. 2004, A\&A, 414, 677

Wilkin, F. P., \& Akeson, R. L. 2003, Ap\&SS, 286, 145 